\title{
APPLICATION OF HDMR METHOD TO RELIABILITY ASSESSMENT OF A SINGLE PILE SUBJECTED TO LATERAL LOAD
}

\author{
JERZY BAUER \\ Wrocław University of Technology, Faculty of Geoengineering, Mining and Geology, \\ Department of Mining and Geology, Wybrzeże Wyspiańskiego 27, 50-370 Wrocław, Poland, \\ e-mail: jerzy.bauer@pwr.wroc.pl
}

\author{
Janusz Kozubal, Wojciech PuŁa, Marek WyJAdŁoWski \\ Wrocław University of Technology, Faculty of Civil Engineering, \\ Institute of Geotechnics and Hydroengineering, Department of Engineering Foundations, \\ Wybrzeże Wyspiańskiego 27, 50-370 Wrocław, Poland, \\ e-mail: wojciech.pula@pwr.wroc.pl; marek.wyjadlowski@pwr.wroc.pl; janusz.kozubal@pwr.wroc.pl
}

\begin{abstract}
The paper presents an application of High Dimensional Model Representation (HDMR) to reliability assessment of a single pile subjected to lateral load. The purpose is to compare HDMR with some classical method based on response surface technique.

First 3D numerical model of the problem for finite elements computations in the ABAQUS STANDARD program has been presented. The soil model is assumed to be linear elastic. However, contacts between the sidewall and the foundation of the pile and the soil are modelled as Coulomb one with friction and cohesion.

Next the Response Surface Method is briefly reviewed in conjunction with reliability approach.

Then the High Dimensional Model Representation approach is presented. In our approach the HDMR algorithm is based on polynomial of the second degree. Finally the numerical studies have been carried out. The first series of computations demonstrate the efficiency of HDMR in comparison to neural network approach. The second series allows comparison of reliability indices resulting from three different approaches, namely neural network response surface, first-order HDMR and second-order HDMR. It has been observed that for increasing values of the length of the pile reliability indices reach similar values regardless of the method response surface applied.
\end{abstract}

\section{INTRODUCTION}

Many numerical procedures have been developed in order to deal with probabilistic analysis concerning geotechnical problems. One of the widest applicable methods is the Response Surface Method. This method, in general, consists in approximating an unknown function by the known function chosen appropriately. From the viewpoint of reliability the Response Surface Method (RSM) serves well problems where probabilistic evaluations are associated with Finite Element Computations. However, the most important drawback of the RSM is that in the case where four or more random variables are present the computational effort increases rapidly. A recent new approach to reliability computation relies on applying the High Dimensional Model 
Representation (HDMR). The HDMR seems to be quite efficient and powerful method. The method has been developed by researchers in applied mathematics and chemistry. The most important papers were given by Rabitz and Omer [31], Sobol [38], Demiralp [7], Kaya and Kaplana [19], Shorter, Ip and Rabitz [36], Li and Wang [20]. Recently some applications to civil engineering problems have appeared (e.g., [27], [28], [33], [34], [37]). Chowdhury and Rao ([5], [6]) were the first to apply HDMR to geotechnical problems, however their suggestions are limited to slope stability problems. By now no applications to probabilistic assessments in other important branches of geotechnical engineering have been reported.

In the present paper the authors developed a numerical algorithm that solves one of the reliability problems associated with laterally loaded foundation piles by means of HDMR. Efficiency of the proposed algorithm will be examined by comparison with response surface obtained by neural networks (Bauer and Puła [1]). The geotechnical problem under consideration is generally complex and needs FEM analyses to be solved. Therefore, an efficient algorithm to carry out the reliability computations is strongly required. The soil model is assumed to be linear elastic. However, contacts between the sidewall and the foundation of the pile and the soil are modeled as Coulomb one with friction and cohesion. On the basis of the numerical pile-soil model and FEM analyses the HDMR response surface is built, which allows efficient conjunction with the well-established methods of reliability measures evaluation, like FORM/SORM ([17], [39]) or Monte Carlo simulations [22]. At the final stage of the paper a comparison between HDMR response surface and the response surface utilising neural network is carried out.

\section{FORMULATION OF THE PROBLEM}

A single pile subjected to lateral load applied in the pile's head is embedded in a soil layer, as presented in Fig. 1.

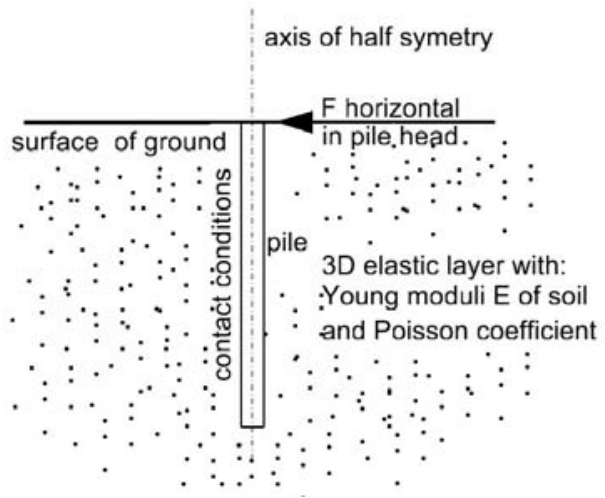

Fig. 1. Graphical scheme of the problem 
If the pile is not perfectly rigid then usually two requirements are essential from a designer point of view. Namely, the constraints state that the horizontal displacement at the pile head from transverse loading as well as the maximum bending moment must not exceed allowable values $u_{\max } M_{\max }$. If the problem is considered as deterministic, one of the classical methods described in the monograph of Reese and Van Impe [35] or paper of Fan and Long [10] can be adopted. It is worth mentioning that in order to get relatively realistic evaluations the problem should be treated as 3-dimensional one. In probabilistic approach soil properties and loads are treated as random variables. Consequently, the head displacement as well as the bending moments are random responses as well. This leads to reliability problem where the probabilities of exceeding the maximal values $u_{\max } M_{\max }$ have to be evaluated. In the framework of the present study only the first requirement, namely the exceeding of value $u_{\max }$ is analysed.

One of the important contributions to addressing a reliability problem of pile subjected to lateral loading in conjunction with the probabilistic approach was presented in the paper by Tandjiria et al [40]. In this paper, the probabilistic analysis of the risk that pile head displacements as well as the maximum bending moments have not exceeded allowable values was made. To enable the computation, of probability measures the response surface method based on the wide-spread $p-y$ curve analysis [25], [26] was applied. In the evaluation, the effect of possible changes in soil properties "with depth" was incorporated. The probability distributions of pile head displacements and of the greatest bending moment have been estimated by means of the Monte Carlo simulation. However, the solutions given by these authors are restricted to a two-dimensional approach and normal distributions of soil properties.

In the paper by Haldar and Sivakumar Babu [14], the properties of soil surrounding the pile have been characterized by non-Gaussian random fields according to the suggestions of Fenton and Griffiths [12], [13]. This approach allows incorporating the spatial variability of soil parameters into reliability computations. The numerical analysis utilises the finite difference method. The probability distributions of pile head displacements as well as the maximal bending moment were evaluated by applying the Monte Carlo method. In this case, solutions were restricted to two dimensions.

In the paper by Chan and Low [4], an analysis in the context of reliability computations was carried out. The procedure presented involves nonlinear pile flexural rigidity with nonlinear $p-y$ curve analysis. The paper investigates two modes of failure: deflection and bending moment. The reliability computations incorporate the FORM [8] approach and use a spreadsheet based numerical procedure. In addition, the response surface method was applied as an alternative supporting the reliability computations.

\section{NUMERICAL MODEL}

A single pile is modelled in three-dimensional space using the ABAQUS STANDARD (GENERAL STATIC) program. Creating the finite element model, the 
existing symmetry plane (Fig. 1) regarding loading and boundary conditions (Fig. 2) is taken into account. Hence, the model consists of two identical half cylinders (Fig. 3). The length of the pile was varied in successive tasks of the problem, namely as $L=\{4.0,8.0,12.0\}^{\mathrm{T}} \mathrm{m}$. The diameter of the circular cross section of the pile has been kept constant as $D=0.50 \mathrm{~m}$.

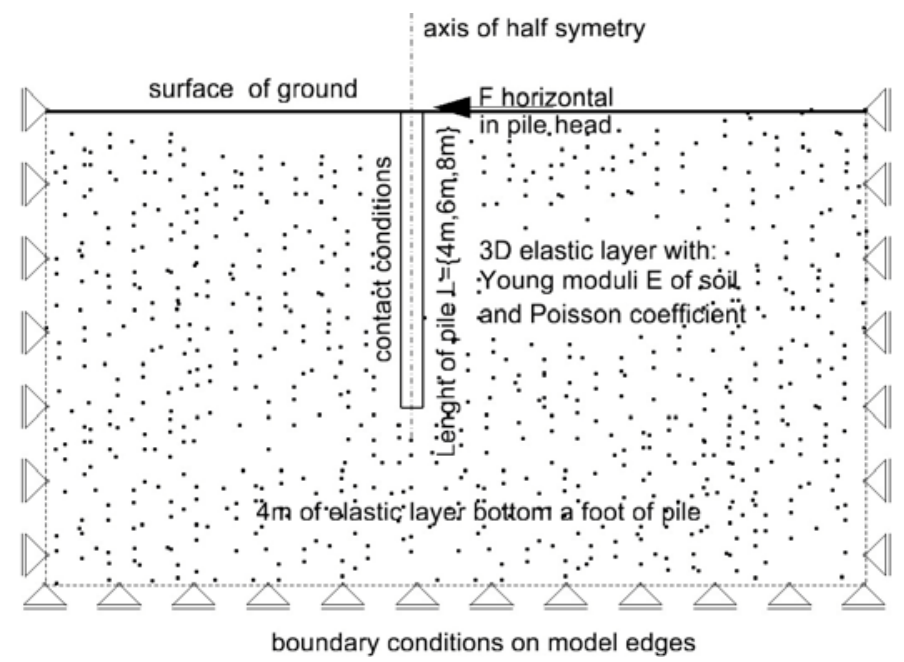

Fig. 2. Numerical model. Symmetry plane cross-section

The width of the model is $40.0 \mathrm{~m}$, the height of the layer in which the pile corresponds to its length $L=\{4.0,8.0,12.0\}^{\mathrm{T}}$, where the thickness of the layer under the foundation of the pile is $4.0 \mathrm{~m}$.

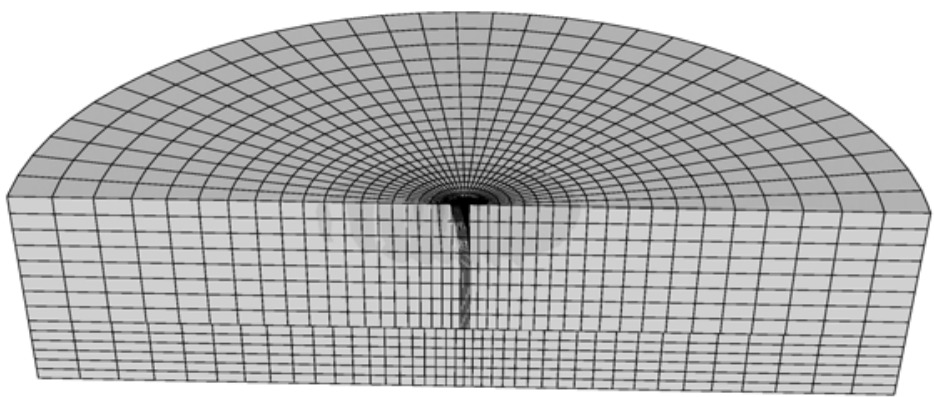

Fig. 3. Numerical model in Finite Elements

The soil is modelled as linear elastic material. The pile head displacements were calculated for the following Young's modulus $E=\{10,20,30,40,50\}^{\mathrm{T}} \mathrm{MPa}$ and a constant Poisson's ratio of $v=0.30$. The pile is made of concrete, with Young's modulus $E_{c}=35 \mathrm{GPa}$. 
The contacts between the sidewall and the foundation of the pile and the soil are modeled as Coulomb one with friction and cohesion. The coefficient of friction equal to the tangent of friction angle was assumed to be $\mu=0.3$. The layer in the contact has the rigidity described with the factors $K_{n n}=K_{s s}=K_{t t}=30 \mathrm{MPa}$, whilst the separation of the surface occurs upon the exceeding of the tension strength related to the cohesion value $c=20 \mathrm{kPa}$.

The calculation is carried out in five steps by the ABAQUS STANDARD (GENERAL STATIC) program, with neglecting geometric non-linearity. Loads are implemented to the head of the pile parallel to the surface successively with resultant five values $F=\{40,55,70,85,100\}^{\mathrm{T}} \mathrm{kN}$. The forces were applied to the horizontal head surface as uniformly distributed loads.

The boundary conditions expressed in displacement take into account the symmetry plane and imply constrains on the side of the model and its bottom. Reduced integration elements - C3D8R were used in the pile with technique to relieve shear locking. These elements use fewer integration points and will often decrease shear locking in certain classes of problems because some terms in the Gauss integration are eliminated. Solution times for these elements are also reduced. In ABAQUS, only the hexahedral elements can use reduced integration [16]. FEM elements are applied to model of soils C3D8 fully integrated linear hexahedral three integration points in each coordinate direction. Therefore, fully integrated linear hexahedral elements have eight integration points. Visually, these points make a smaller cube within the element. The dimensions of the elements are chosen in such a way as to allow a quick calculation for the contact. The nodes adjacent to each part of the model and size of the elements are mutually corresponding. The model consists of about 7500 finite elements. Infinite elements are not used on the sides of the model, as decided by preliminary calculations to check the accuracy of calculation.

To calculate the response surface, 75 computing cycles were performed. The model and the results of calculations in the ABAQUS program are stored in a database format. The access to information can be done from different levels. A script in the Python language is used in this study. The script is responsible for the change of material features, model and forces, control calculations and after receiving a set of results, it carries out transfer of results to the TXT file report.

Calculations were performed in ABAQUS 6.9-1, based on the grant of the Wrocław Centre for Networking Supercomputing (wcss.wroc.pl).

\section{THE RESPONSE SURFACE METHOD}

\subsection{LIMIT STATE FUNCTION}

A typical question within the framework of serviceability limit states of pile can be formulated as follows. What is the chance of exceeding a given threshold value $u_{\max }$ by the head displacement of a pile. The value of $u_{\max }$ can be given for same special 
requirements of a structures a whole or can be postulated by codes. If we assume that some subsoil parameters are random variables, then as a measure of reliability the following probability can be considered

$$
p_{F}=P\left(U\left(x_{1}, x_{2}, \ldots, x_{N}\right)>u_{\max }\right),
$$

where $U\left(x_{1}, x_{2}, \ldots, x_{N}\right)=U(\mathbf{x})$ is the horizontal displacement of a pile head affected by a set of random parameters $x_{1}, x_{2}, \ldots, x_{N}$ like subsoil model parameters, loads, geometrical properties, etc., as well as some other properties considered as deterministic. The values of $U$ are usually given by means of some numerical procedures as FEM, for example. The function $U$ is called the limit state function. The probability (1) is known as probability of failure. In engineering computation usually alternative measure of reliability (namely the reliability index $\beta$ is utilized). The beta index is associated with probability $p_{f}$ by the relationship

$$
p_{F}=\Phi(-\beta)
$$

where $\Phi$ is the standard normal cumulative distribution function. Suggestions regarding values of beta in various situations are given by ISO code [18].

\subsection{RESPONSE SURFACE}

Examining the head displacement of a pile the explicit form of the function $U$ is rarely known. The values of $U$ are usually given by means of some numerical procedures as FEM, for example. To enable further reliability computations of FEM results the Response Surface Method (RSM) can be adopted.

The RSM applied to numerous fields of knowledge is exhaustively described in a number of monographs, e.g., Box and Draper ([29], [2]). In general, this method consists in approximating an unknown function by the known function chosen appropriately. This approximation can be based on the results of experiments and also on the results of numerical computations, e.g., results obtained by means of the FEM. In the case of numerical computations, a relationship between the model parameters $x_{1}$, $x_{2}, \ldots, x_{N}$, which are introduced as input data, and the values obtained as output data $y=f\left(x_{1}, x_{2}, \ldots, x_{N}\right)$ is defined. Roughly establishing such a relationship allows us to replace a troublesome numerical procedure with a simple analytical relationship, which helps to forecast a response of the model being analysed in the input set.

The RSM was adapted to the reliability analyses of engineering structures in the 1980s by Rackwitz ([32], [9]) and Faravelli [11]. In order to simplify reliability computations, rather simple functions such as polynomials of the second degree are often used [3]. An efficient algorithm for the purpose of settlement analysis has been proposed by Bauer and Pula [1]. Numerical procedures of approximation of unknown function $U$ are diverse. In any case algorithms based on regression models may be used. 


\subsection{NON-LINEAR REGRESSION METHOD \\ IN RESPONSE SURFACE MODELLING}

Generally speaking, the form of the function $U$ can be described by the following non-linear regression model

$$
\mathrm{E}(U)=f(\mathbf{x}, \mathbf{b})+e r r,
$$

where $\mathrm{E}(U)$ is an expected value of $U ; \mathbf{x}$ is a vector of $N$ independent variables (number of parameter in equation (1)), $\mathbf{b}$ is a vector of $k$ unknown parameters of regression model $f$ and err is a random variable describing the error of estimation of function $U$.

The components of vector $\mathbf{b}$ are determined by the process of minimizing the sum of the squares of differences between given quantities $U_{i}$ and predicted quantities

$$
\Psi=\sum_{i}^{n}\left(U_{i}-\hat{U}_{i}\right)^{2}
$$

for the set of $n$ data: $\left(U_{i}, x_{i l}\right), i=1,2, \ldots, n, l=1,2, \ldots, N$.

Random variable err of the estimation error takes the zero expected value and its standard deviation is equal to

$$
S_{\text {err }}=\sqrt{\frac{\Psi_{\min }}{n-k}} .
$$

There exist two classic methods of finding the minimum of function $\Psi$, namely the linearization method and the steepest gradient method [23], [24].

\subsection{NEURAL NETWORK AS RSM}

As an alternative approach utilising neural network can be adopted. Here a neural network with a hyperbolical activation function is suggested. If a continuous function is approximated then the three-layered neural network with one hidden layer containing necessary number of neurons could give a satisfactory result. Neural networks allow approximation in cases of large variability intervals of independent variables preserving sufficient accuracy. To get required accuracy, according to Kolmogorov's theorem, the number of neurons in the hidden layer is increased. In examples presented in the next section a three-layered neural network of three inputs in the first layer representing three independent random variables will be used. The hidden layer consists of two neurons with hyperbolic tangent as the activation functions [15]. The summation layer has a hyperplane expression as an additional term. The architecture of the applied network is presented in Fig. 4. 


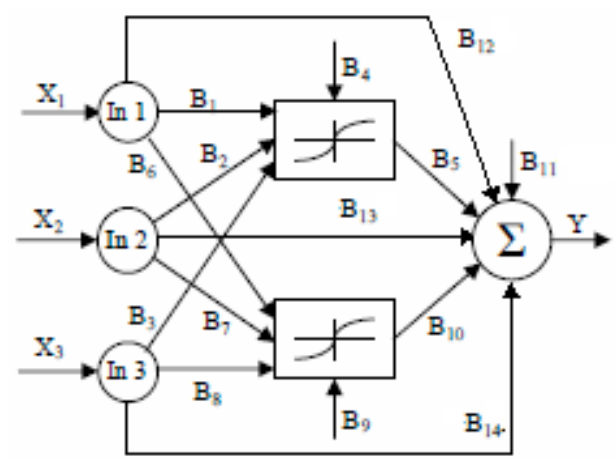

Fig. 4. The architecture of the neural network applied.

The network consists of three layers. Two neurons are in the hidden layer

As a consequence of the applied neural network the following response surface will be obtained

$$
\begin{gathered}
y(\mathbf{x})=b_{5} \tanh \left(b_{1} x_{1}+b_{2} x_{2}+b_{3} x_{3}+b_{4}\right)+b_{10} \tanh \left(b_{6} x_{1}+b_{7} x_{2}+b_{8} x_{3}+b_{9}\right) \\
+b_{11}+b_{12} x_{1}+b_{13} x_{2}+b_{14} x_{3} .
\end{gathered}
$$

In the above equation, $x_{1}$ corresponds to the loading of the pile head $F, x_{2}$ is the length of the pile $L$ and $E$ stands for Young's modulus. The above network includes fourteen weights that have to be found in the learning process. Instead of classical method of learning a network by the algorithm of backwards error propagation the weights $b_{1} \ldots b_{14}$ were evaluated in the iteration process of non-linear regression method based on Marquartd algorithm [23].

\subsection{HIGH DIMENSIONAL MODEL REPRESENTATION AS RSM}

The guiding idea of application of High Dimensional Model Representation (HDMR) method to function not being an explicit one, is reduction of necessary numerical calculations, indispensable in designating its values with simultaneous maintenance of the quality of the fit. HDMR allows describing the model with numerous variables, which has already been proved on functions tested by the authors. The method for three chosen independent variables $N=3$ has been applied in this paper. HDMR is featured by a significant reduction of the number of computations in relation to classical approximations of classified functions with several variables, which is going to be explained further. The HDMR method is based on unknown function development, also presented as a surface response function of the structure in the task: $g(\mathbf{x})$, where $\mathbf{x}=\left\{x_{1}, x_{2}, \ldots, x_{N}\right\}^{\mathrm{T}}$ represents vector of $N$ variables. Function $g(\mathbf{x})$ can be expanded according to the following equations ([6]) 


$$
\begin{gathered}
g(\mathbf{x})=g_{0}+\sum_{i=1}^{N} g_{i}\left(x_{i}\right)+\sum_{1 \leq i_{1}<i_{2} \leq N} g_{i_{1} i_{2}}\left(x_{i_{1}}, x_{i_{2}}\right) \\
+\ldots+\sum_{1 \leq i_{1}<\ldots<i_{k} \leq N} g_{i_{1} i_{2} \ldots i_{k}}\left(x_{i_{1}}, x_{i_{2}}, \ldots, x_{i_{k}}\right)+g_{12 \ldots N}\left(x_{1}, x_{2}, \ldots, x_{N}\right),
\end{gathered}
$$

where:

- $g_{0}$ is the value of function $g$, at the central point $\mathbf{c}$ being already defined at the start, e.g., coordinates of $\mathbf{x}$ may be expected values of random variables in our task. The term $g_{0}$ is called the zeroth-order or the mean response.

- $g_{i}\left(x_{i}\right)$ is single variable function, where solely $x_{i}$ changes and the other coordinates remain constant and equal to the ones of a central point. The total of all components following and including $g_{0}$ are called the first order expansion.

- $g_{i_{1} i_{2}}\left(x_{i_{1}}, x_{i_{2}}\right)$ is a function of acting variables $x_{i_{1}}$ and $x_{i_{2}}$ only. Both are varied. Other coordinates are equal to corresponding coordinates of central point. The first three components in equation (7) constitute the second-order expansion.

- $g_{i_{1} i_{2} \ldots i_{k}}\left(x_{i_{1}}, x_{i_{2}}, \ldots, x_{i_{k}}\right)$ describes the cooperative effects of increasing numbers of input variables. They are the subsequence of $k$ elements from all variables. The first $k+1$ components in equation (7) create the $k$ th-order expansion.

- The last element of equation (7) stands for any residual dependence of all input variables equal $g(x)$ minus the $k$ th-order.

In the following study we have applied an algorithm for choosing particular ranges of variable that cover (interesting in the task) area. Additionally, we have selected the reference point in equation $\mathbf{c}=\left\{c_{1}, c_{2}, \ldots, c_{N}\right\}^{T}$. Equations describing the first three components are

$$
\begin{gathered}
g_{0}=g(\mathbf{c}), g_{0}=g(\mathbf{c}), \\
g_{i}\left(x_{i}\right)=g\left(x_{i}, c^{i}\right)-g_{0}, \\
g_{i_{1} i_{2}}\left(x_{i_{1}}, x_{i_{2}}\right)=g_{i_{1} i_{2}}\left(x_{i_{1}}, x_{i_{2}}, c^{i_{1} i_{2}}\right)-g\left(x_{i_{1}}\right)-g\left(x_{i_{2}}\right)-g_{0} .
\end{gathered}
$$

As a result, the first-order and second-order expansions are

$$
\begin{gathered}
g(\mathbf{x})=g_{0}+\sum_{i=1}^{N}\left(g\left(x_{i}, c^{i}\right)-g(\mathbf{c})\right)+\theta_{2}, \\
g(\mathbf{x})=g_{0}+\sum_{i=1}^{N}\left(g\left(x_{i}, c^{i}\right)-g(\mathbf{c})\right) \\
+\sum_{1 \leq i_{1}<i_{2} \leq N}\left(g_{i_{1} i_{2}}\left(x_{i_{1}}, x_{i_{2}}, c^{i_{1} i_{2}}\right)-g\left(x_{i_{1}}\right)-g\left(x_{i_{2}}\right)-g(\mathbf{c})\right)+\theta_{3},
\end{gathered}
$$


where $\Theta_{2}$ and $\Theta_{3}$ are residual errors. After some mathematical transformations one gets

$$
\begin{gathered}
g(\mathbf{x})=\sum_{i=1}^{N} g\left(x_{i}, c^{i}\right)-(N-1) g(\mathbf{c})+\theta_{2}, \\
g(\mathbf{x})=\sum_{1 \leq i_{1}<i_{2} \leq N}\left(g_{i_{1} i_{2}}\left(x_{i_{1}}, x_{i_{2}}, c^{i_{i} i_{2}}\right)\right)-(N-2) \sum_{i=1}^{N} g_{i}\left(x_{i}, c^{i}\right)+\frac{1}{2}(N-1)(N-2) g(\mathbf{c})+\theta_{3} .
\end{gathered}
$$

Hence, taking into account equations (13) and (14), the first order approximation is given by

$$
\tilde{g}(\mathbf{x})=\sum_{i=1}^{N} g\left(x_{i}, c^{i}\right)-(N-1) g(\mathbf{c})
$$

and the second order by

$$
\widetilde{g}(\mathbf{x})=\sum_{1 \leq i_{1}<i_{2} \leq N}\left(g_{i_{1} i_{2}}\left(x_{i_{1}}, x_{i_{2}}, c^{i_{1} i_{2}}\right)\right)-(N-2) \sum_{i=1}^{N} g_{i}\left(x_{i}, c^{i}\right)+\frac{1}{2}(N-1)(N-2) g(\mathbf{c}) .
$$

The comparison of approximate function values of (13) and (15) gives us residual error $\theta_{2}$, and of (14) and (16) $\theta_{3}$.

The first step consists in estimating value $g(\mathbf{c})$. Subsequently, all values in crosssections $g\left(x_{i}, c^{i}\right)$ need to be calculated. The calculation points, $\mathbf{x}^{j}, j=1, \ldots, n$, should be spread out regularly and symmetrically with respect to the central point $\mathbf{c}$. Therefore, coordinates of calculation points can be given by

$$
x_{i}^{j}=\min \left(x_{i}\right)+j \frac{\left(\max \left(x_{i}\right)-\min \left(x_{i}\right)\right)}{n}, \quad j=1, \ldots, n .
$$

We have to remember that our goal is to find an approximate form of the implicit function $g(\mathbf{x})$. Within this study polynomials of the second degree are utilised as approximate explicit function, however, other forms of explicit function can be used alternatively (some examples are given in Osada [30]).

The first-order components for cross-section $i$ are

$$
g\left(x_{i}, c^{i}\right) \approx \Phi_{i}\left(x_{i}, \alpha_{i}\right)
$$

where the functions $\Phi_{i}$ are fitted by means of the least square method

$$
\Phi_{i}\left(x_{i}, \alpha_{i}\right)=\sum_{k=1}^{p} \alpha_{i, k} x_{i}^{k-1} .
$$


In this equation, $\alpha_{i}$ are coefficients of approximate polynominals of degree $p$ (in our case $p=2$ ). In consequence, the first-order approximate function with respect to equation (15) is defined by

$$
\mathrm{G}^{I}(x)=\sum_{i=1}^{N} \Phi_{i}\left(x_{i}, \alpha_{i}\right)-(N-1) g_{0}
$$

In the case of involving two variables approximate functions $\Phi_{i_{1} i_{2}}\left(x_{i_{1}}, x_{i_{2}}, \alpha_{i i_{1}}\right)$ are of the form (see equation (16))

$$
\mathrm{G}^{\mathrm{II}}(x)=\sum_{\substack{i_{1}=1, i_{2}=1 \\ i_{1}<i_{2}}}^{N} \Phi_{i_{1} i_{2}}\left(x_{i_{1}}, x_{i_{2}}, \alpha_{i_{1} i_{2}}\right)-(N-2) \sum_{i=1}^{N} \Phi_{i}\left(x_{i}, \alpha_{i}\right)+\frac{(N-1)(N-2)}{2} g_{0} .
$$

In most of the cases we have considered the approximation of the second-order only due to high fit precision.

\section{COMPARISON OF THE METHODS}

In the previous section we described the numerical model and based on it we made calculations. We used the RSM in the three cases: the neural network and HDMR with the first and second-order.

In the case of three dimensional models, if the response surface incorporating neural network has to be established, it is necessary to carry out 75 runs of FEM computations. With increasing number of random variables the quantity of computing cycles increases exponentially. In complicated tasks this is a serious obstacle in the description of the phenomenon.

For determining the elements of HDMR method it is necessary to designate 13 points by numerical calculations in the case of first-order fitting and 61 points in the case of second-order fitting. The difference in the number of necessary calculations increases with increasing dimension of the task, in favour of the HDMR method. The increase, however, is not as rapid as in the neural network case. The results of comparative calculations are summarized in Table 1.

Table 1

Comparison of the quality adjustment methods presented in the article

\begin{tabular}{|l|c|c|c|}
\hline $\begin{array}{c}\text { Method of response } \\
\text { surface determination }\end{array}$ & $\begin{array}{c}\text { Number of } \\
\text { necessary points }\end{array}$ & $\begin{array}{c}\text { Standard } \\
\text { deviation } \boldsymbol{s}_{\text {err }}(4)\end{array}$ & $\begin{array}{c}R^{2}[\%] \text { determination } \\
\text { coefficient }\end{array}$ \\
\hline Neural networks (6) & 75 & 0.334 & 99.6 \\
\hline HDMR first-order (HD_3) & 11 & 1.550 & 92.1 \\
\hline HDMR second-order (21) & 43 & 0.269 & 99.8 \\
\hline
\end{tabular}


High efficiency of HDMR of the second-order is observed. For almost double the smaller number, in relation to the neural network approximation, the data needed for calculations of the numerical experiment we get a better quality match. It should be noted that both the standard deviation and coefficient of determination were established with the use of all the 75 points obtained from FEM.

Table 2

Summary of distributions of parameter values for reliability calculations

\begin{tabular}{|l|c|c|c|}
\hline \multicolumn{1}{|c|}{ Variable } & $\begin{array}{c}\text { Type of } \\
\text { distribution }\end{array}$ & Mean value $\mu$ & Standard deviation $\sigma$ \\
\hline Young's modulus $E$ & lognormal & $30 \mathrm{MPa}$ & $1.5 \mathrm{MPa}$ \\
\hline Force $F$ & normal & $70 \mathrm{kN}$ & $7 \mathrm{kN}$ \\
\hline
\end{tabular}

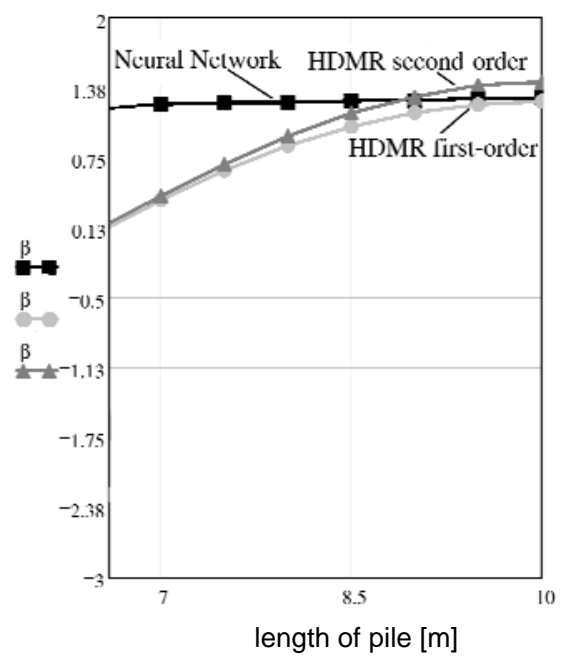

Fig. 5. Influence of the pile's length on reliability index $\beta$ value.

Other variables take values: $E=30 \mathrm{MPa}, F=70 \mathrm{kN}$.

Ordinates denote the pile's length. The graph is created for three methods: neural networks, first-order HDMR and second-order HDMR

For the task described the probability of failure has been evaluated according to equation (1) and reliability index $\beta$ by means of equation (2), using the three abovedescribed adjustment and probabilistic assumptions depicted in Table 2.

Computations were carried out using Monte Carlo procedure with sample size equal to $10^{6}$. The results are shown in Fig. 5. It is worth noting that for increasing values of the length of the pile reliability indices reach similar values regardless of the method response surface applied. 


\section{CONCLUSIONS}

This paper presents an application of HDMR numerical method to evaluate the reliability of pile subjected to horizontal loads and comparison with classical the Response Surface Method based of neural network technique. It has be shown that HDMR can successfully replace the classical method. This modification gives a much greater efficiency than traditionally used algorithms of approximation of response surface, e.g., using artificial neural networks. Particularly valuable is the ability to include greater number of random variables than in classical approach.

When geotechnical task is solved by finite element method, the number of calculations needed may make it very difficult or even impossible to estimate the reliability index. Therefore, improving the computational efficiency gained by using HDMR method is important. As a result of the calculations a good coincidence of reliability indices for the first-order and the second-order HDMR is demonstrated. In the case of the problem under consideration, the first-order HDMR the computational effort, when only 11 points are used, gives the similar values of reliability index as more accurate, but more laborious, the mapping of the second-order.

The task under consideration has required to build a three-dimensional numerical model. For this purpose it was necessary to develop computational algorithms and perform their testing and comparison with the methods previously used by the authors.

Calculations were made using the Python programming language which ensures coherence in the program code ABAQUS where FEM calculations are carried out. This solution allows us to parameterise, analyse and compile the results in a single development environment. The results highlight the possibility of using HDMR method in other problems associated with the estimation of measures reliability in geotechnics.

\section{REFERENCES}

[1] BAUER J., PUŁA W., Neural network supported response surface method with respect to reliability computations in geotechnics, Studia Geotechnica et Mechanica, 2000, 22, No. 3-4, 103-115.

[2] Box G.P., Draper N.R., Empirical Model-Building and Response Surface, J. Wiley \& Sons, New York, 1996.

[3] BuCher C.G., BouRgund U., A fast and efficient response surface approach for structural reliability problems, Structural Safety, 1990, 7, 57-66.

[4] Chan C.L., Low B.K., Reliability Analysis of Laterally Loaded Piles Involving Nonlinear Soil and Pile Behavior, J. Geotech. and Geoenvir. Engrg., 2009, 135(3), 431-443.

[5] Chowdhury R., Rao B., Hybrid High Dimensional Model Representation for reliability analysis, Comput. Methods Appl. Mech. Engrg., 2009, 198, 753-765.

[6] Chowdhury R., Rao B., Probabilistic Stability Assessment of Slopes Using High Dimensional Model Representation, Computers and Geotechnics, 2010. 
[7] Demiralp M., High Dimensional Model Representation and its application varieties, Tools for Mathematical Methods, Mathematical Research, St-Petersburg, 2003, Vol. 9, 146-159.

[8] Ditlevsen O., Madsen H.O., Structural reliability Methods, John Wiley \& Sons, Chichester, 1996.

[9] ENGElund S., RACKWITZ R., Experiences with experimental design schemes for failure surface estimation and reliability, Proc. 6th Speciality Conf. Probabilistic Mechanics and Structural and Geotechnical Reliability, Denver, 1992, 252-255.

[10] FAn C.C., Long J.H., Assessment of existing methods for predicting soil response for laterally loaded piles in sand, Computers and Geotechnics, 2005, 32, 274-289.

[11] FARAVELLI L.A., A response surface approach for reliability analysis, Journal of the Engineering Mechanics Division, ASCE, 1989, 115(12), 2763-2781.

[12] FeNTON G.A., GRIFFITHS D.V., Bearing capacity prediction of spatially random c- $\varphi$ soils, Canadian Geotechnical Journal, 2003, 40(1), 545.

[13] Fenton GA., Griffiths D.V., Risk Assessment in Geotechnical Engineering, John Wiley \& Sons, New York, 2008.

[14] Haldar S., Sivakumar M., Babu G.LS., Effect of soil spatial variability on the response of laterally loaded pile on undrained clay, Computers and Geotechnics, 2008, 35, 537-547.

[15] Hecht-Nielson R., Neurocomputing, Addison Wesley, Amsterdam, 1991.

[16] HibBitt D., KARLSSON B., Sorensen P., ABAQUS Keywords Manual Version 6.3 Hibbitt, Karlsson \& Sorensen, Inc., USA, 2002.

[17] Hohenbichler M., Gollwitzer S., Kruse W., Rackwitz R., New light on first and second-order reliability methods, Structural Safety, 1987, 4, 267-284.

[18] ISO 2394:2000. General principles on reliability of structures. International Standard.

[19] Kaya H., Kaplana M., Saygina H., A recursive algorithm for finding HDMR terms for sensitivity analysis, Computer Physics Communications, 2004, 158, 106-112.

[20] Li G., WANG S.W., RABITZ H., Global uncertainty assessments by high dimensional model representation (HDMR), Chemical Engineering Science, 2002, Vol. 57, 4445-4460.

[21] Li G., RABITZ H., Regularized random-sampling high dimensional model representation (RSHDMR), Journal of Mathematical Chemistry, March 2008, Vol. 43, No. 3.

[22] Magiera R., Models and methods of mathematical statistics, GiS, Wrocław, 2007.

[23] MARQUARDT D.W., An algorithm for least-squares estimation of non-linear parameters, J. Soc. Indust. Appl. Math., June 1963, 11, No. 2.

[24] MARQUARDT D.W., Least-squares estimation of non-linear parameters computer code, NLIN2, Distribution No. 309401, IBM Share Library, August 1966.

[25] Matlock H., Correlations for design of laterally loaded piles in soft clay, Proc., Offshore Technology Conference, Houston, Texas, 1970, 577-594.

[26] McClelland B., Focht J.A., Soil modulus for laterally loaded piles, Transactions of the ASCE, 1958, 123, 1049-1086.

[27] Mukherjee D., RaO B., Prasad A.-M., Global Sensitivity Analysis of Unreinforced Masonry Structure Using High Dimensional Model Representation, Engineering Structures, April 2011, Vol. 33, No. 4, 1316-1325,.

[28] Mukherjee D., Rao B., Prasad A., Cut-HDMR Based Fully Equivalent Operational Model for Analysis of Unreinforced Masonry Structure, Sadhana, 2012.

[29] Myers R.H., Montgomery D.C., Response Surface Methodology Process and Product Optimisation Using Design Experiments, John Wiley \& Sons, New York, 1995.

[30] OSADA E., Geodesy, Wroclaw University of Technology, Wrocław, 2002.

[31] RABitz H., OMER F., Alis General foundations of high-dimensional model representations, Journal of Mathematical Chemistry, 1999, 25, 197-233 197. 
[32] RACKWITz R., Response surfaces in structural reliability, Berichte zur Zuverlässigkeitstheorie der Bauwerke, H. 67, LKI, Technische Universität München, 1982.

[33] Rao B., ChOwdhURY R., Factorized high dimensional model representation for structural reliability analysis, Engineering Computations International Journal for Computer-Aided Engineering and Software, 2008, Vol. 25, No. 8, 708-738.

[34] Rao B., Chowdhury R., Probabilistic Analysis Using High Dimensional Model Representation and Fast Fourier Transform, International Journal for Computational Methods in Engineering Science and Mechanics, 2008, 9, 342-357.

[35] ReESE LC., Van ImPE W.F., Single Piles and Pile Groups Under Lateral Loading, Balkema, Rotterdam, 2001.

[36] Shorter J.A., Ip P.C., Rabitz H., An Efficient Chemical Kinetics Solver Using High Dimensional Model Representation, J. Phys. Chem. A, 1999, Vol. 103, 7192-7198.

[37] Sivakumar M., Rao B., Satishkumar S.R., The Effect of Pressure induced Hoop Stress on Biaxially Loaded Through wall Cracked Cylindrical Structures - A Strain Based Method, Applied Mechanics and Materials, 2012, Vol. 110-116, No. 4, 1525-1530.

[38] SoвоL I., Theorems and examples on high dimensional model representation, Reliability Engineering and System Safety, 2003, 79, 187-193.

[39] STRUREL, A Structural Reliability Analysis Program System, COMREL \& SYSREL, Users Manual, RCP Consultant, Munich, 1995.

[40] TANDJIRIA V., TEH C.I., LOW B.K., Reliability analysis of laterally loaded piles using response surface methods, Structural Safety, 2000, 22(4), 335-355. 